\title{
The Transcription Factor ATF4 Promotes Expression of Cell Stress Genes and Cardiomyocyte Death in a Cellular Model of Atrial Fibrillation
}

\author{
Johanna K. Freundt, ${ }^{1,2}$ Gerrit Frommeyer, ${ }^{1}$ Fabian Wötzel, ${ }^{3}$ Andreas Huge, ${ }^{4}$ \\ Andreas Hoffmeier, ${ }^{5}$ Sven Martens, ${ }^{5}$ Lars Eckardt, ${ }^{1}$ and Philipp S. Lange $\mathbb{D}^{1}$ \\ ${ }^{1}$ Department of Cardiovascular Medicine, Division of Electrophysiology, University Hospital Münster, Germany \\ ${ }^{2}$ Institute of Physiology II, University of Münster, Germany \\ ${ }^{3}$ Department of Pathology, University Hospital Münster, Germany \\ ${ }^{4}$ Core Facility Genomik, Medical Faculty, Westfälische Wilhelms-Universität Münster, Germany \\ ${ }^{5}$ Department of Cardiac and Thoracic Surgical, University Hospital Münster, Germany \\ Correspondence should be addressed to Philipp S. Lange; philippsebastian.lange@ukmuenster.de
}

Received 25 November 2017; Revised 29 March 2018; Accepted 15 April 2018; Published 29 May 2018

Academic Editor: Rui Liu

Copyright ( $\odot 2018$ Johanna K. Freundt et al. This is an open access article distributed under the Creative Commons Attribution License, which permits unrestricted use, distribution, and reproduction in any medium, provided the original work is properly cited.

\begin{abstract}
Introduction. Cardiomyocyte remodelling in atrial fibrillation $(\mathrm{AF})$ has been associated with both oxidative stress and endoplasmic reticulum (ER) stress and is accompanied by a complex transcriptional regulation. Here, we investigated the role the oxidative stress and ER stress responsive bZIP transcription factor ATF4 plays in atrial cardiomyocyte viability and AF induced gene expression. Methods. HL-1 cardiomyocytes were subjected to rapid field stimulation. Forced expression of ATF4 was achieved by adenoviral gene transfer. Using global gene expression analysis and chromatin immunoprecipitation, ATF4 dependent transcriptional regulation was studied, and tissue specimen of AF patients was analysed by immunohistochemistry. Results. Oxidative stress and ER stress caused a significant reduction in cardiomyocyte viability and were associated with an induction of ATF4. Accordingly, ATF4 was also induced by rapid field stimulation mimicking AF. Forced expression of wild type ATF4 promoted cardiomyocyte death. ATF4 was demonstrated to bind to the promoters of several cell stress genes and to induce the expression of a number of ATF4 dependent stress responsive genes. Moreover, immunohistochemical analyses showed that ATF4 is expressed in the nuclei of cardiomyocytes of tissue specimen obtained from AF patients. Conclusion. ATF4 is expressed in human atrial cardiomyocytes and is induced in response to different types of cell stress. High rate electrical field stimulation seems to result in ATF4 induction, and forced expression of ATF4 reduces cardiomyocyte viability.
\end{abstract}

\section{Introduction}

Atrial fibrillation (AF) is the most common arrhythmia in industrialized countries with an increasing burden of morbidity. However, despite the overwhelming clinical relevance of AF, fundamental mechanisms governing the maintenance and perpetuation of atrial fibrillation remain poorly understood. Known pathophysiological mechanisms include but are not limited to oxidative stress, abnormal $\mathrm{Ca}^{2+}$ homeostasis, ion channel dysfunction, and microRNA mediated dysregulation [1]. However, key molecular and electrophysiological changes leading to $\mathrm{AF}$ and to disease progression have not yet been fully elucidated. As a consequence of our limited understanding of the complex pathophysiology of AF, the prevention and treatment strategies of this arrhythmia still need to be optimized.

Atrial remodeling is characterized by complex structural and electrical changes leading to atrial dilatation and atrial fibrosis thereby promoting conduction slowing, spontaneous depolarizations and action-potential duration prolongation. Cardiomyocyte apoptosis is assumed to play an important role in atrial remodeling and disease progression $[2,3]$. In fact, the ageing heart is experiencing a constant loss of cardiomyocyte (estimated $0.5-1 \%$ per year $[1,4]$ ), and fibrous tissue often 
replaces cardiomyocytes undergoing cell death. Moreover, age is one of the major risk factors for the development of $\mathrm{AF}[4]$.

Oxidative stress, calcium overload, and endoplasmic reticulum (ER) stress seem to play important roles in AF and AF induced atrial remodeling $[5,6]$. In many cells, including cardiomyocytes, the expression of genes that can mitigate the consequences of oxidative stress and ER stress is precisely coordinated by a synergistic network of stress-sensing signaling cascades [7-9]. Specifically, ER stress caused by calcium overload and other stressors and aberrantly elevated levels of oxidants can trigger the transcriptional induction of a number of adaptive pathways [10]. This cellular stress response is tightly controlled by a family of stress-responsive transcription factors. Among these transcription factors, the activating transcription factor $4(\mathrm{ATF} 4) / \mathrm{cAMP}$ response element binding protein 2 might be particularly important. While being constitutively expressed only at low concentrations, ATF4 can be rapidly induced under particular cell-stress conditions [11]. ER stress leads to decreased translation of most cellular mRNAs; paradoxically, the mRNA that encodes activator of ATF4 is translated more efficiently. Increased levels of ATF4 serve important roles as a transcriptional inducer of a certain ER stress response genes, which assist in recovery from the stress [12]. During the prosurvival phase of the ER stress response ATF4 induces numerous genes involved in resolution of the ER stress, such as genes that encode amino acid transporters and ER resident chaperones [13]. However, after prolonged ER stress, continued ATF4 expression mediates the upregulation of genes that contribute to programmed cell death. For example, ATF4 induces the transcription factor C/EBP homologous protein $[14,15]$, which induces numerous proapoptotic proteins, including GADD34 [16], and Tribbles-related protein 3 [17]. Moreover, $\mathrm{CHOP}$ regulates expression of several $\mathrm{Bcl} 2$ family members $[15,18,19]$. ATF4 binds to the promoter regions of several different target genes, including many involved in ER stress and redox control. In fibroblasts, ATF4 has an important role in the cellular response to amino acid depletion, oxidative stress, and endoplasmic reticulum stress and helps to balance redox homeostasis. More specifically, ATF4-deficient fibroblasts have been shown to be prone to death when exposed to different types of stresses, including oxidative stress and amino acid deprivation [20]. To get a more conclusive insight in the mechanism of AF-induced myocyte remodeling, we used a cell culture based model of atrial fibrillation. Rapid pacing of cultured atrial-derived myocytes HL-1 mimics the phenotypic feature of tachycardia-induced atrial cardiomyocyte remodelling in vivo $[21,22]$. Here, we show that ATF4 expression impairs atrial cardiomyocyte survival in an in vitro model of atrial fibrillation. Moreover, we demonstrate that ATF4 is expressed at relevant levels in atrial cardiomyocytes in vivo and might play an important role in atrial remodeling in response to atrial fibrillation.

\section{Materials and Methods}

A detailed description of the methods can be found in the supplements.
2.1. Immunohistochemistry. Atrial appendage tissue was obtained from 17 individual patients undergoing cardiac surgery with $(n=9)$ and without atrial fibrillation $(n=9)$. Patient characteristics are described in Table 2. The study was approved by the University of Münster Ethical Committee and Institutional Review Board. Therefore, it has been performed in accordance with the ethical standards laid down in the 1964 Declaration of Helsinki and its later amendments. All patients gave their written informed consent for the histological examination. ATF4 was detected with a commercially available antibody (rabbit polyclonal anti-ATF4, 1:200, LS-B3517, LSBio). ATF4 positive nuclei were counted in representative fields of all patient specimen. mRNA was extracted and subjected to real-time PCR.

2.2. HL-1 Cell Culture and Pacing. The murine cardiomyocyte cell line HL-1 was kindly provided by Dr. Claycomb, Lousiana State University $[23,24]$. The cell line was cultured in Claycomb medium (Sigma) supplemented with $0.1 \mathrm{mM}$ norepinephrine (Sigma), $2 \mathrm{mM}$ L-glutamine (Biochrom), $100 \mathrm{U} / \mathrm{mL}$ penicillin (Biochrom), $100 \mu \mathrm{g} / \mathrm{mL}$ streptomycin (Biochrom), and $10 \%$ fetal bovine serum (Sigma). The myocytes were cultured in flasks coated with $5 \mu \mathrm{g} / \mathrm{ml}$ fibronectin (Sigma) and $0.02 \%$ gelatin (Sigma), in a $5 \% \mathrm{CO}_{2}$ atmosphere at $37^{\circ} \mathrm{C}$. They were split at full confluence with $0.05 \% / 0.02 \%$ trypsin-EDTA (Biochrom) 1:2 or 1:3 and media were changed every 24 to 48 hours. Rapid pacing of HL-1 cells was performed with the C-Pace EP Culture Stimulation System (IonOptix). To induce tachycardia, HL-1 myocytes were cultured on coverslips in $35 \mathrm{~mm}$ dishes. At $80 \%$ confluence the coverslips were embedded into the C-Dish between the carbon electrodes and fresh medium was added. After $1 \mathrm{~h}$ at $37^{\circ} \mathrm{C}$ the cells were subjected to an electrical field stimulation $(1 \mathrm{~Hz}, 10 \mathrm{~ms}, 20 \mathrm{~V}$, biphasic waveform) for $1 \mathrm{~h}$ and then to a modified electrical field stimulation $(4 \mathrm{~Hz}, 10 \mathrm{~ms}, 20 \mathrm{~V})$ for $20 \mathrm{~h} .1 \mu \mathrm{M}$ Thapsigargin was added to the HL-1 medium and incubated for $18 \mathrm{~h}$. $0-0.1 \mu \mathrm{l} / \mathrm{ml} \mathrm{H}_{2} \mathrm{O}_{2}$ was incubated for $6 \mathrm{~h} .2$ and $4 \mu \mathrm{g} / \mathrm{ml}$ tunicamycin were added to the HL-1 medium and incubated for $5 \mathrm{~h}$.

2.3. Isolation of Cardiac Fibroblasts. The cardiac fibroblasts were isolated from ventricles from adult C57BL/6 mice.

2.4. Infection of HL-1 Cells and Fibroblasts with Adenovirus. Cells were infected with virus particles containing GFP (vVQ-pEF-GFP-mycTag-K-NpA), ATF4 wild type (vVQpEF-ATF4wt-mycTag-K-NpA), or ATF4 $\Delta$ RK (vVQ-pEFATF4 $\Delta$ RK-mycTag-K-NpA) (ViraQuest Inc.) as described elsewhere [25].

2.5. Preparations of Protein Lysates and Western Blot Analyses. Western blot membranes were incubated with the following primary antibodies: anti- $\beta$-actin (sc-1616, 1:1000, Santa Cruz) and anti-ATF4 (sc-200x, 1:5000, Santa Cruz) and secondary antibody anti-goat $(1: 5000$, Santa Cruz) and antirabbit $(1: 1000$, Dako). Complete western blots are shown in Supplemental Figure S2.

2.6. Quantitative Real-Time PCR Analysis. Real-time PCR analysis with cDNA from HL-1 cells was performed using 
TaqMan Gene Expression Master Mix (Applied Biosystems) with the following primers: ATF4 (Mm00515325_g1, Applied Biosystems) and ß-actin (Mm00607939_s1, Applied Biosystems). Real-time PCR analysis from human tissue was performed using Sybr Green qPCR Master Mix (Roboklon) with the following primers: ATF4 (forward primer: TCAAACCTCATGGGTTCTCC, reverse primer: GTGTCATCCAACGTGGTCAG) and $ß$-actin (forward primer: ATTGCCGACAGGATGCAGAA, reverse primer: ACATCTGCTGGAAGGTGGACAG).

2.7. Microarray Analysis. HL-1 cells were infected with Adenovirus containing a GFP, ATF4wt, or ATF4 4 RK construct. After $48 \mathrm{~h}$, cells were rapidly paced for $20 \mathrm{~h}$. Biotin-labeled cRNA was hybridized on Illumina MouseWG-6 v2.0 expression BeadChips. In the Illumina GenomeStudio Software 2011.1, raw data were normalized using the quantile algorithm. Differential gene expression was assessed on the basis of grouped replicates and thresholds for expression ratios or, alternatively, for both expression ratios and statistical significance employing the $t$-test model, based on standard deviations between biological replicates. Filtering of genes was performed using sorting and autofiltering functions in MS Excel ( $p$ value $\leq 0.05)$. Functional annotation analysis was carried out using DAVID 6.7.

2.8. Chromatin Immunoprecipitation (ChIP) Assay. HL-1 cells were infected with Adenovirus containing a GFP, ATF4wt, or ATF $4 \Delta \mathrm{RK}$ construct. DNA binding proteins were crosslinked for $10 \mathrm{~min}$ in $0.5 \%$ formaldehyde. The HL-1 cells were lysed and sonicated at $40 \%$ amplitude for $10 \mathrm{~s}$ on, $20 \mathrm{~s}$ off, and 20 cycles on ice. Each sample was incubated with anti-myc antibody (9B11, Cell Signalling) overnight at $4^{\circ} \mathrm{C}$. The ChlPDNA was added to a library preparation (NEBNext ChIP Seq Library Preparation), and a single read sequencing was performed (Core Facility Genomik University of Münster) using the NextSeq 500 System. The algorithm MACS2 was used for identifying transcript factor binding sites. Functional annotation analysis was carried out using DAVID 6.7. The software package BETA was used to compare genes from the ChIP Assay and the Microarray analysis. Filtering of genes was performed using sorting and autofiltering functions in MS Excel (fold change $\geq 1.2$ and $\leq 0.83$ ).

2.9. Statistical Analysis. Two-sample independent Student's $t$-tests were used to compare the means of two groups (SPSS Version 22, SPSS). Differences with a $p$ value of $\leq 0.05$ were considered to be statistically significant.

\section{Results}

Both oxidative stress and ER stress play key roles in the atrial remodeling process in atrial fibrillation. ATF4 is a key transcription factor mediating cellular gene expression changes in response to different types of cell stress. Therefore, we initially hypothesized that ATF4 could be induced by both oxidative stress and ER stress in atrial cardiomyocytes. We initiated our study with an analysis of ATF4 expression on mRNA and protein level in the atrial cardiomyocyte cell line HL-1 in response to both oxidative stress and ER stress. We analyzed the expression of ATF4 in response to oxidative stress induced by treatment with hydrogen peroxide. Accordingly, oxidative stress heightened ATF4 expression level on both the mRNA and protein level (Figures 1(a) and 1(b)). ER stress in atrial cardiomyocytes was induced by thapsigargin, an agent that raises the cytosolic calcium concentration thereby mimicking calcium overload, a condition that has been associated with endoplasmic reticulum stress. Treatment of cardiomyocytes with thapsigargin caused an increase of ATF4 mRNA and ATF4 protein expression (Figures 1(a) and 1(c)). Correspondingly, treatment with tunicamycin, a well characterized ER stress inducing agent, also led to an increased level of ATF4 expression (Supplemental Figure 1). Both oxidative stress and ER stress were associated with a decrease in cell viability as measured by MTT assay (Figure 1(e)). Thus, we hypothesized that ATF4 expression might also be elevated in response to atrial fibrillation, a condition that has been associated both with oxidative stress and ER stress. In order to study the response to atrial fibrillation in atrial cardiomyocytes, we used an established cellular model of AF, in which HL-1 cardiomyocytes are subjected to rapid field stimulation. Accordingly, rapid field stimulation led to an induction of ATF4 mRNA, an increased ATF4 expression on the protein level, and decreased cell viability (Figures 1(a), 1(d), and 1(e)).

To further investigate the role of ATF4 in cardiomyocytes, we continued our study analyzing the effect of forced expression of ATF4 in atrial cardiomyocytes. Adenoviral vectors were used to overexpress wild type ATF4 in HL-1 cells. For a negative control, we used GFP or a dominant negative ATF4 mutant (ATF4 $\triangle \mathrm{RK}$ ) (Figure 2(a)). In general, cytotoxicity was raised by electrical stimulation of HL-1 cells (Figure 2(b)). Forced expression of ATF4 further increased cytotoxicity in electrically stimulated HL-1 cardiomyocytes compared to HL-1 cells infected with GFP or with ATF4 $\Delta$ RK. Accordingly, an MTT assay showed a reduced viability of the electrically stimulated cells and a further reduction of viability by expression of wild type ATF4 (Figure 2(c)).

In order to decipher the gene expression changes associated with electrical stimulation and ATF4 expression, a global analysis of ATF4 target genes was carried out by overexpression of ATF4, ATF4 $\triangle \mathrm{RK}$, or GFP in electrically stimulated and nonstimulated HL-1 cardiomyocytes and subsequent microarray analysis. In nonstimulated cardiomyocytes, overexpression of ATF4 led only to a minor change in gene expression (Figure 3(a)). 30 genes were upregulated and 2 genes were downregulated by ATF4 compared to cells infected with GFP-vector. The ATF4 mutant did not display regulatory properties in nonstimulated cardiomyocytes (comparison of ATF $4 \Delta \mathrm{RK}$ versus GFP overexpressing cardiomyocytes). However, in electrically stimulated cardiomyocytes, ATF4 caused a relevant change in gene expression (comparison of ATF4wt stimulated versus GFP stimulated). 240 genes were upregulated and 149 genes were downregulated as a consequence of ATF4 overexpression in stimulated cells compared to electrically stimulated cells with GFP overexpression. The most profound gene expression differences were observed 


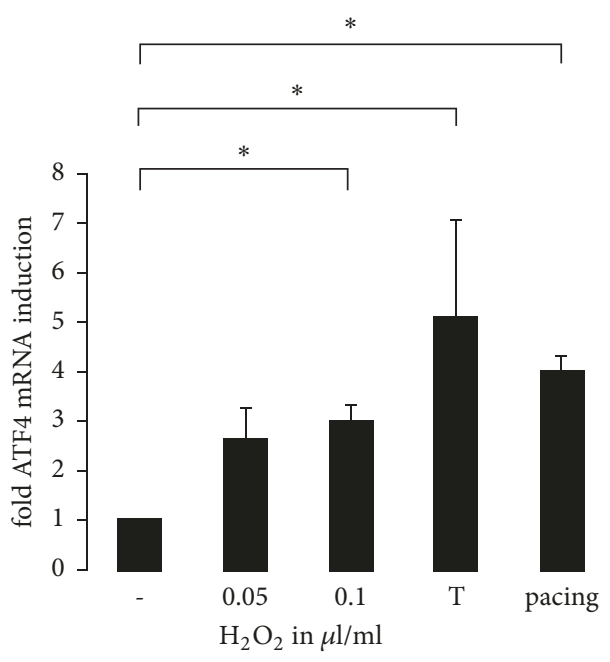

(a)

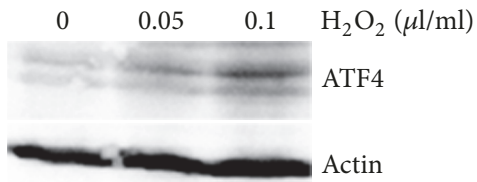

(b)

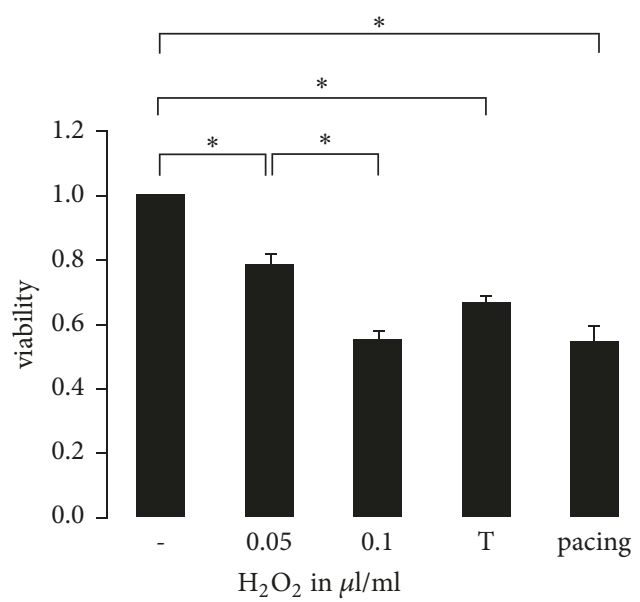

(e)

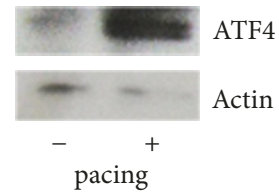

(d)

FIGURE 1: ATF4 expression in $\mathrm{H}_{2} \mathrm{O}_{2}$ and thapsigargin treated cardiomyocytes and in electrically stimulated cardiomyocytes. (a) Real-time PCR of ATF4 mRNA expression in cultured HL-1 cardiomyocytes in response to treatment with $0,0.05$, or $0.1 \mu \mathrm{l} / \mathrm{ml}_{2} \mathrm{O}_{2}(n=3)$, real-time PCR of ATF4 mRNA expression in cultured cardiomyocytes in response to treatment with $1 \mu \mathrm{M}$ thapsigargin $(n=3)$, and real-time PCR of cardiomyocytes stimulated electrically with $4 \mathrm{~Hz}$ for $20 \mathrm{~h}(n=3)$. Representative western blots showing protein expression of ATF4 in response to $\mathrm{H}_{2} \mathrm{O}_{2}$ treatment (b) and in response to thapsigargin treatment (c). (d) Cardiomyocytes were electrically stimulated with $4 \mathrm{~Hz}$ for $20 \mathrm{~h}$ and ATF4 protein expression was detected. (e) MTT assay displaying viability of cardiomyocytes treated with $\mathrm{H}_{2} \mathrm{O}_{2}(n=3), 1 \mu \mathrm{M}$ thapsigargin $(n=3)$, and paced HL-1 cardiomyocytes $(n=5)$. * indicates $p<0.05$.

when comparing ATF4 overexpressing, electrically unstimulated cardiomyocytes to ATF4 overexpressing, and electrically stimulated cardiomyocytes. Electrical stimulation in ATF4 overexpressing cardiomyocytes caused an upregulation of 2947 genes and a downregulation of 2429 genes (Figure 3(a) and Table 1(a)). Pathway enrichment analysis in this dataset (Figure 3(b), upper panel) revealed that electrical stimulation in ATF4 overexpressing cardiomyocytes led to a pattern of gene expression characteristic of the cellular response to ER stress, oxidative stress, inflammation, and cell death including the upregulation of genes that are involved in the MAPK signaling pathway. ATF3, Dusp2, and Fos belong to this group of genes (Table 1(a)). ATF3 is a member of the mammalian activation transcription factor/cAMP responsive element-binding (CREB) protein family of transcription factors and is known to be induced in response to different types of cell stress; Dusp2 and Fos are genes of the MAPK signaling pathway.

In electrically stimulated cells, ATF4 overexpression led to a regulation of the p53 signaling pathways and TNF/stress related signaling (Figure 3(b), middle panel). On the contrary, ATF4 overexpression in electrically unstimulated HL1 cardiomyocytes mainly influenced the expression of genes involved in amino acid biosynthesis (Figure 3(b), lower panel).

Thus, ATF4 seems to favor the expression of a number of defined target genes that are associated with cell stress and stress adaptive pathways. These target genes may be either induced directly by binding of ATF4 to its promoters or subsequently as a consequence of ATF4 induced adaptive pathways. In order to identify ATF4's primary target genes that are regulated directly by binding of ATF4 to its promoters, ATF4 and its non-DNA-binding mutant (ATF4 $\Delta$ RK) as well as GFP were overexpressed in HL-1 cardiomyocytes, followed by Chromatin Immunoprecipitation and then fed onto a "ChIP-on-chip" assay. ATF4wt and ATF4 $\Delta$ RK overexpression led to a regulation of genes with a significantly different expression level (fold change $\geq 1.2$ or $\leq 0.83$ ) in ATF4wt and ATF4 $\triangle \mathrm{RK}$ overexpressing cardiomyocytes in comparison to the GFP dataset. These genes were contrasted in a heatmap (Figure 4(a)). Several genes were regulated by ATF4wt as well as by ATF4 $\Delta$ RK. In total, 2015 genes were subject of regulation compared to GFP overexpressing cardiomyocytes (Venn diagram in Figure 4(b) (upper panel)). In order to identify genes that were specifically bound by ATF4 and to rule out nonspecific binding, 90 genes were 
TABLE 1: Genes regulated by ATF4. (a) List of top 45 genes being up- or downregulated in stimulated (S) HL-1 cardiomyocytes overexpressing ATF4wt (corresponding to Figure 3(a)). (b) List of top 48 genes that are regulated in cardiomyocytes overexpressing ATF4wt but not in HL-1 cells with overexpressing ATF4 $\Delta$ RK detected by ChIP assay (corresponding to the grey area in Figure 4(b)). (c) Genes being up- or downregulated by stimulation in cardiomyocytes overexpressing ATF4wt detected by microarray and enrichment in ChIP assay of HL-1 cardiomyocytes overexpressing ATF4wt (corresponding to the grey area in Figure 4(c)).

(a)

\begin{tabular}{|c|c|c|}
\hline \multicolumn{3}{|c|}{ Array ATF4wt stim versus GFP stim } \\
\hline Gene Symbol & fold change & $1 / p$ value \\
\hline Saa3 & 16.40 & $2.78 \mathrm{E}-34$ \\
\hline Avil & 10.58 & $6.34 \mathrm{E}-07$ \\
\hline Gsta3 & 3.39 & $3.98 \mathrm{E}-03$ \\
\hline Gadd45a & 3.22 & $7.62 \mathrm{E}-22$ \\
\hline Serpinf1 & 2.96 & $1.67 \mathrm{E}-17$ \\
\hline Csn3 & 2.69 & $2.78 \mathrm{E}-34$ \\
\hline $\operatorname{Lcn} 2$ & 2.69 & $4.88 \mathrm{E}-04$ \\
\hline Clec2e & 2.59 & $1.67 \mathrm{E}-17$ \\
\hline Dgat2 & 2.51 & $1.43 \mathrm{E}-07$ \\
\hline Gulo & 2.50 & $2.33 \mathrm{E}-05$ \\
\hline Hdc & 2.44 & $1.73 \mathrm{E}-02$ \\
\hline Sprr2f & 2.41 & $2.38 \mathrm{E}-04$ \\
\hline Colec1l & 2.40 & $3.51 \mathrm{E}-03$ \\
\hline Iyd & 2.22 & $7.11 \mathrm{E}-03$ \\
\hline Iah1 & 2.21 & $2.07 \mathrm{E}-05$ \\
\hline Aqp9 & 2.17 & $1.29 \mathrm{E}-06$ \\
\hline $\mathrm{Tg}$ & 2.13 & $2.49 \mathrm{E}-02$ \\
\hline Cox6a2 & 2.12 & $6.01 \mathrm{E}-08$ \\
\hline Was & 2.11 & $2.86 \mathrm{E}-05$ \\
\hline Agpat9 & 2.08 & $4.97 \mathrm{E}-03$ \\
\hline Gp5 & 2.08 & $4.65 \mathrm{E}-03$ \\
\hline Reep6 & 2.07 & $1.93 \mathrm{E}-03$ \\
\hline ATF4 & 1.51 & $3.35 \mathrm{E}-03$ \\
\hline Ifit3 & 0.30 & $1.01 \mathrm{E}-02$ \\
\hline Irf7 & 0.38 & $1.57 \mathrm{E}-07$ \\
\hline Gbp3 & 0.40 & $1.31 \mathrm{E}-02$ \\
\hline Iigp2 & 0.43 & $2.06 \mathrm{E}-07$ \\
\hline Vwa5a & 0.43 & $3.75 \mathrm{E}-10$ \\
\hline Igtp & 0.43 & $2.71 \mathrm{E}-02$ \\
\hline Myadm & 0.44 & $6.31 \mathrm{E}-03$ \\
\hline Lgals3bp & 0.45 & $1.25 \mathrm{E}-04$ \\
\hline Parp14 & 0.45 & $8.69 E-03$ \\
\hline Trim41 & 0.45 & $1.77 \mathrm{E}-02$ \\
\hline Galnt11 & 0.45 & $6.09 \mathrm{E}-03$ \\
\hline Batf2 & 0.47 & $4.82 \mathrm{E}-06$ \\
\hline Lgals9 & 0.49 & $1.72 \mathrm{E}-02$ \\
\hline Eif2ak2 & 0.50 & $4.89 \mathrm{E}-04$ \\
\hline Oaslb & 0.50 & $3.76 \mathrm{E}-03$ \\
\hline Gbl & 0.51 & $7.16 \mathrm{E}-06$ \\
\hline Irgb10 & 0.53 & $1.95 E-06$ \\
\hline Tap1 & 0.53 & $3.22 \mathrm{E}-02$ \\
\hline Pln & 0.53 & $1.46 \mathrm{E}-05$ \\
\hline Ifi35 & 0.54 & $7.86 \mathrm{E}-06$ \\
\hline Psmb9 & 0.54 & $4.91 \mathrm{E}-04$ \\
\hline Cxcl1 & 0.56 & $3.19 \mathrm{E}-05$ \\
\hline Wdr6 & 0.56 & $5.56 \mathrm{E}-04$ \\
\hline
\end{tabular}


(b)

\begin{tabular}{|c|c|}
\hline \multicolumn{2}{|c|}{ ChIP ATF4wt w/o ATF4dRK } \\
\hline Gene Symbol & $-\log p$ value \\
\hline Stk40 & 38.47 \\
\hline Ddit3 & 35.56 \\
\hline Arhgef33 & 35.47 \\
\hline ВC071253 & 33.68 \\
\hline Herpud1 & 31.72 \\
\hline AK018753 & 29.82 \\
\hline $\mathrm{Abcc} 8$ & 27.51 \\
\hline AK140265 & 26.10 \\
\hline Gm13889 & 24.81 \\
\hline Gm10222 & 24.27 \\
\hline DQ539915 & 23.56 \\
\hline Rp9 & 22.95 \\
\hline $\operatorname{Cox} 2$ & 22.34 \\
\hline Atpase6 & 22.29 \\
\hline $\mathrm{E} 2 \mathrm{f} 4$ & 21.63 \\
\hline Cytb & 21.28 \\
\hline Atf3 & 20.76 \\
\hline Aars & 20.29 \\
\hline Zxdc & 20.02 \\
\hline Cebpb & 17.44 \\
\hline Wnt3a & 17.33 \\
\hline Rpn2 & 17.25 \\
\hline Atpase6 & 17.22 \\
\hline Ddr2 & 16.66 \\
\hline Siah2 & 16.34 \\
\hline Trim14 & 13.19 \\
\hline Apbb2 & 13.05 \\
\hline Eif1 & 11.93 \\
\hline Abhd11 & 11.57 \\
\hline Trmt12 & 11.27 \\
\hline Psat1 & 11.21 \\
\hline Pknoxl & 10.68 \\
\hline Med30 & 10.34 \\
\hline Abcc8 & 10.15 \\
\hline Mthfd2 & 10.08 \\
\hline Gars & 10.04 \\
\hline Slc25a26 & 9.88 \\
\hline Nupr1 & 9.53 \\
\hline Nars & 9.07 \\
\hline Fbln5 & 9.06 \\
\hline Trib3 & 8.14 \\
\hline Crip2 & 8.03 \\
\hline Asap1 & 7.97 \\
\hline Gm16197 & 7.93 \\
\hline Cebpg & 7.81 \\
\hline
\end{tabular}


(c)

\begin{tabular}{lcc}
\hline & $\begin{array}{c}\text { Array ATF4wt and ChIP ATF4wt } \\
\text { Gene Symbol }\end{array}$ & $1 / p$ value \\
\hline Asns & 1.66 & 0.0002 \\
Trib3 & 1.49 & 1.0000 \\
Aars & 1.38 & 0.0410 \\
Psat1 & 1.37 & 1.0000 \\
Aldh18a1 & 1.34 & 1.0000 \\
Mthfd2 & 1.34 & 0.1265 \\
Jdp2 & 1.33 & 0.8275 \\
Eif2s2 & 1.31 & 0.1495 \\
Rhbdd1 & 1.28 & 1.0000 \\
Atad2 & 1.25 & 1.0000 \\
Herpud1 & 1.23 & 1.0000 \\
Nupr1 & 1.20 & 1.0000 \\
S100a6 & 0.79 & 1.0000 \\
\hline
\end{tabular}

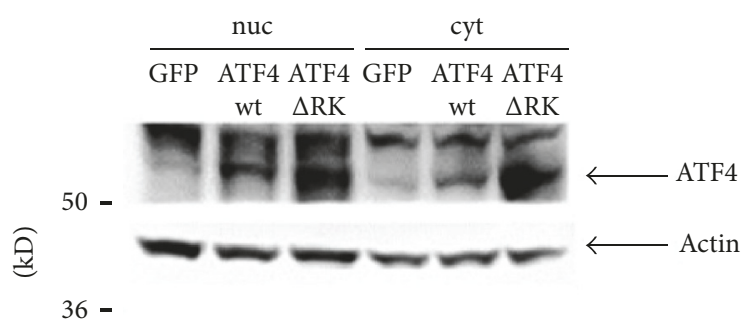

(a)

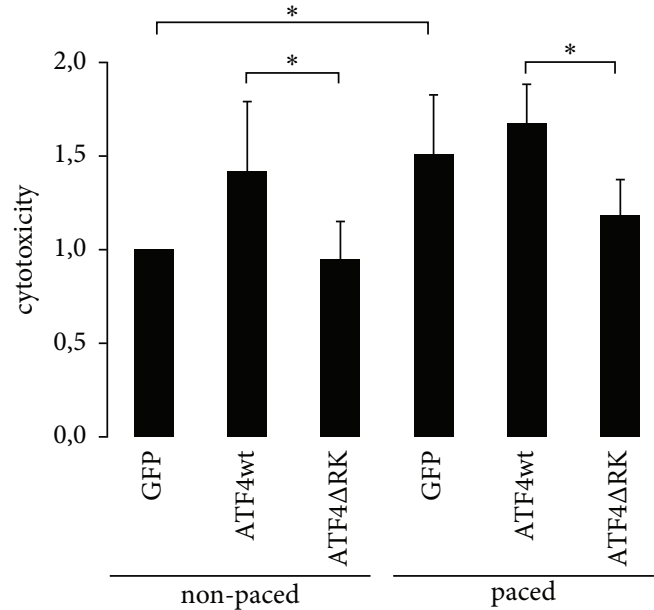

(b)

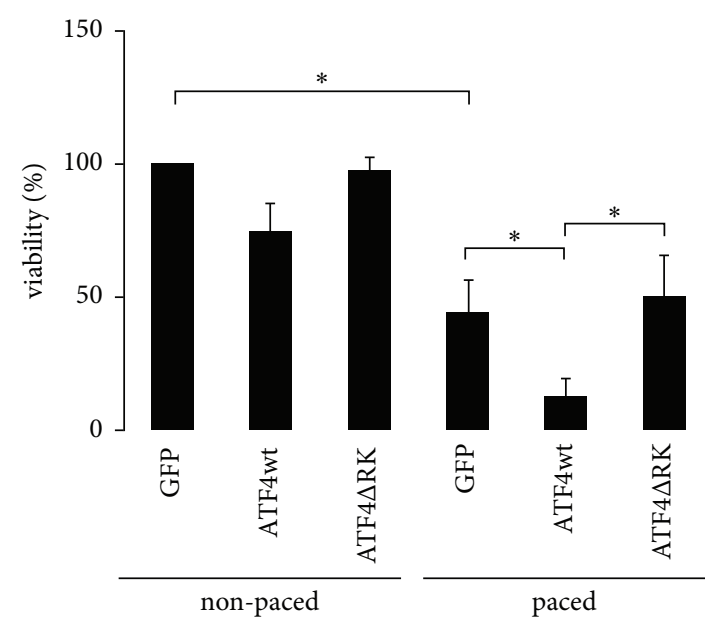

(c)

FIGURE 2: Effect of pacing on viability in cardiomyocytes overexpressing ATF4. (a) Representative western blots showing nuclear (nuc) and cytosolic (cyt) protein expression of ATF4 in cardiomyocytes overexpressing GFP, ATF4wt, or ATF4 $\Delta$ RK. GFP, ATF4wt, or ATF4 $\Delta$ RK were overexpressed in cardiomyocytes followed by pacing. Cytotoxicity was measured by LDH-assay $(n=3)(b)$ and viability was measured by MTT-Assay $(n=4)(\mathrm{c}), *$ indicates $p<0.05$.

identified that were bound by ATF4wt and did not appear in the ATF $4 \Delta \mathrm{RK}$ dataset (grey area in the Venn diagram of Figure 4(b)). In the pathway enrichment analysis, this group of genes can be attributed to molecular pathways of amino acid biosynthesis, endoplasmic reticulum stress, and cell death (Figure 4(b), lower panel). Trib3, Ddit3, and ATF3 belong to this group (Table 1(b)). Trib3 is a well characterized ATF4 target gene. The putative protein kinase binds to ATF4 and inhibits its transcriptional activation activity. Ddit3 plays an essential role in the response to a wide variety of cell 


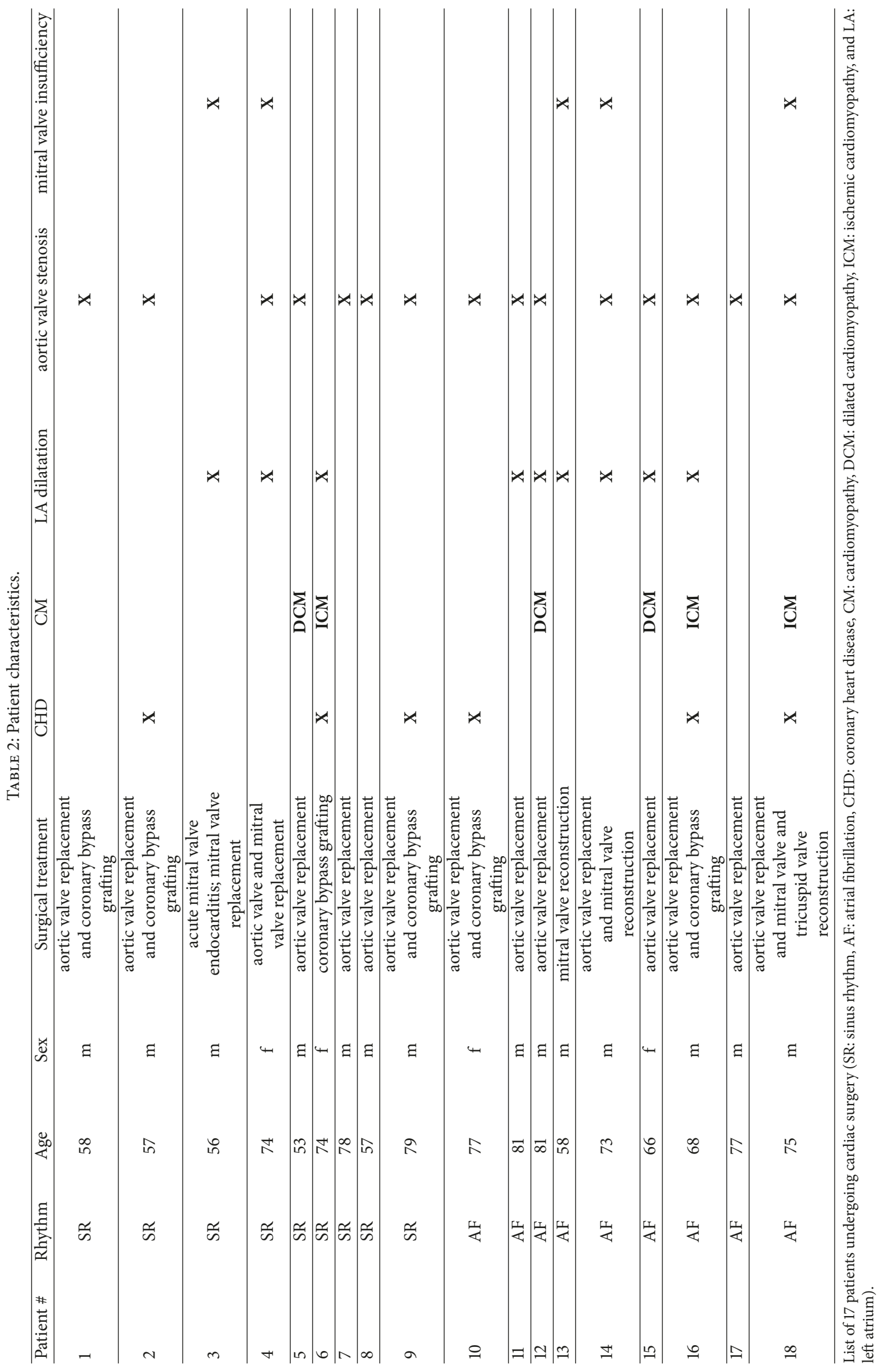




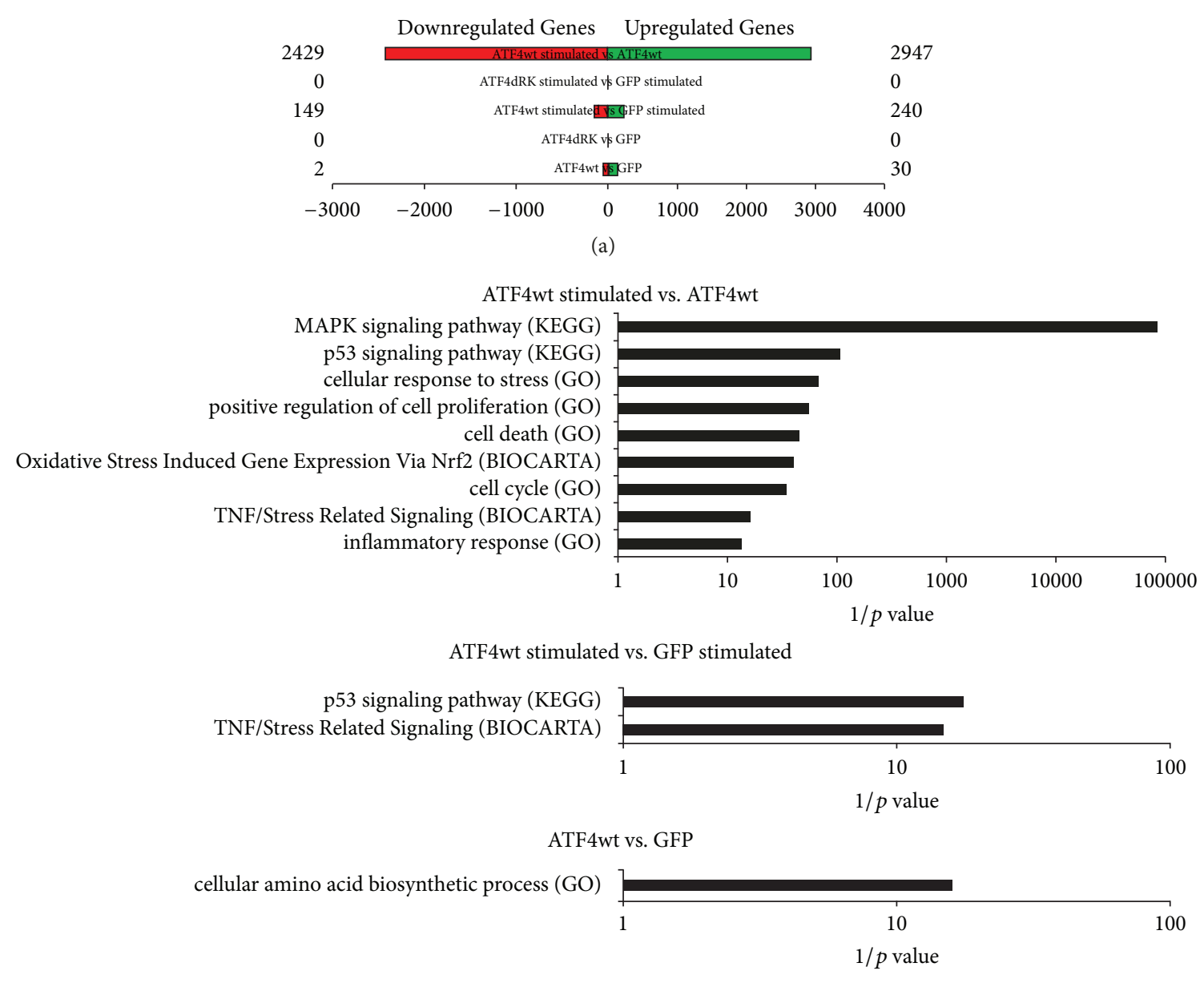

(b)

Figure 3: Gene expression microarray analysis. (a) The number of genes compared to control (GFP) that are up- (green) and downregulated (red) in cardiomyocytes overexpressing ATF4 wild type (wt) or ATF4 $\Delta$ RK with and without electrical stimulation (fold change $\geq 1.2$ or $\leq 0.83$, and $p$ value $\leq 0.05$ ). (b) Pathways enriched in electrically stimulated ATF4wt overexpressing HL-1 cardiomyocytes versus unstimulated ATF4wt overexpressing HL-1 cardiomyocytes (upper panel), electrically stimulated ATF4wt overexpressing cardiomyocytes versus stimulated GFP overexpressing cardiomyocytes (middle panel), and ATF4wt versus GFP overexpressing cardiomyocytes (lower panel).

stresses and has been shown to induce apoptosis in response to ER stress [26].

Next, we identified genes that possess an ATF4wt DNAbinding site (ChIP-on-chip assay) and are regulated in stimulated cardiomyocytes with ATF4wt overexpression compared to nonstimulated cardiomyocytes with ATF4wt overexpression (mRNA microarray). 30 analog genes were found. This group of genes is involved in the ER stress response and apoptosis (Figure 4(c), lower panel, and Table 1(c)). ATF3 and Ddit3 were unregulated in this group of genes.

In order to clarify ATF4's role in atrial fibrillation in vivo, the study was complemented with an immunohistological analysis of atrial tissue obtained from patients undergoing cardiac surgery (Table 2). Atrial tissue obtained from patients with a documented history of atrial fibrillation $(n=9)$ was compared to tissue obtained from patients without atrial fibrillation $(n=9)$. ATF4 could be detected with a nuclear localization predominantly in cardiomyocytes (Figure 5(a)). An elevated number of ATF4 positive cardiomyocytes was present in tissue specimen obtained from patients with atrial fibrillation compared to patients in sinus rhythm (Figure 5(b)); however, human ATF4 mRNA levels of tissue specimen detected by real-time PCR did not significantly differ between the groups.

In comparison to cardiomyocytes, cardiac fibroblasts did not display a strong ATF4 immunoreactivity. However, in order to assess the functional role of ATF4 expression in cardiac fibroblasts and in the development of fibrosis, we analyzed the effects of ATF4 overexpression in cultured cardiac fibroblasts. Fibroblasts were infected with GFP, ATF4wt, and ATF $4 \Delta$ RK adenoviruses (Figure 6(a)). Subsequently, cell proliferation was assessed by a BrdU assay. The proliferation of murine fibroblasts was markedly reduced by ATF4wt overexpression compared to GFP overexpression. In ATF4 $\Delta \mathrm{RK}$ 


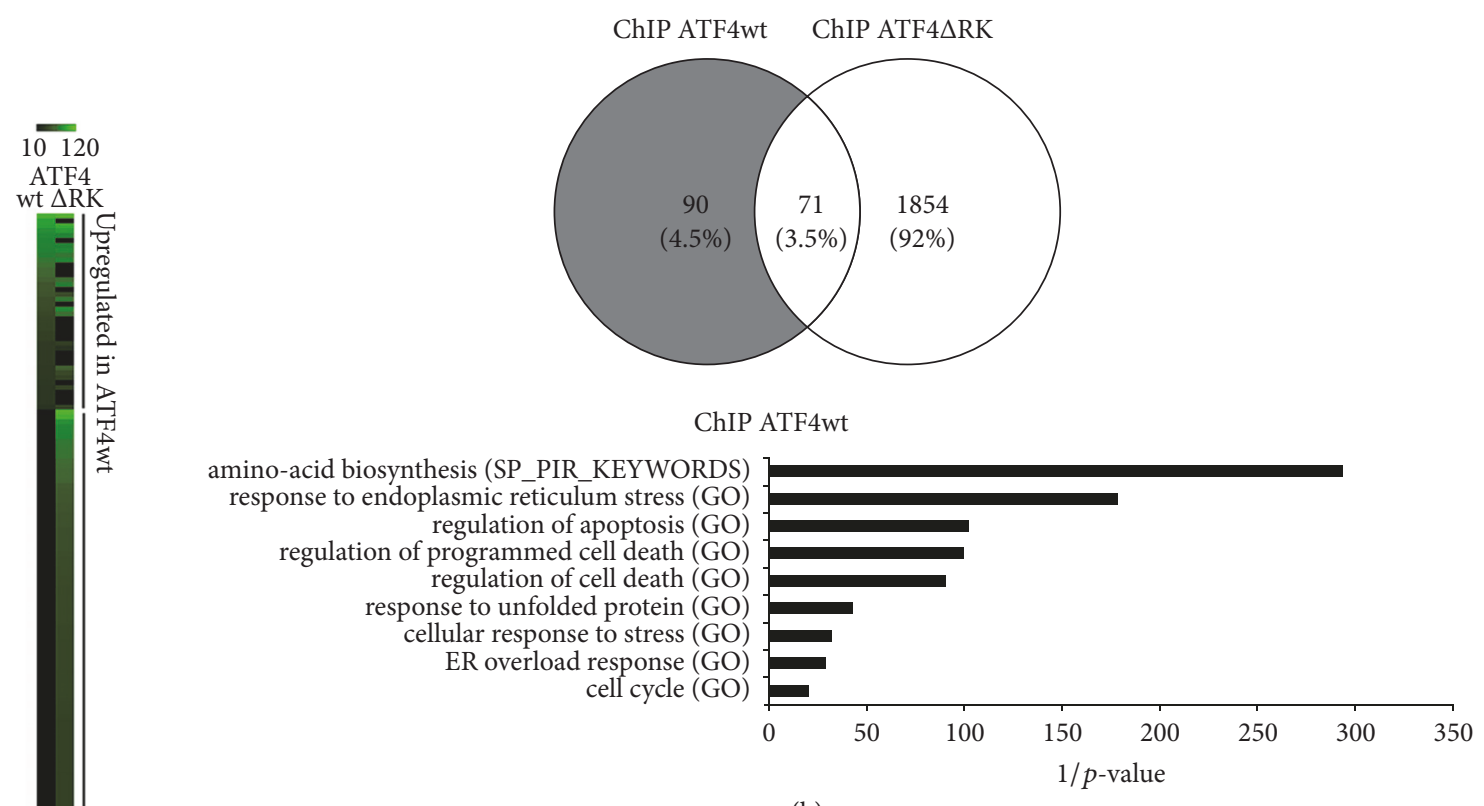

(b)

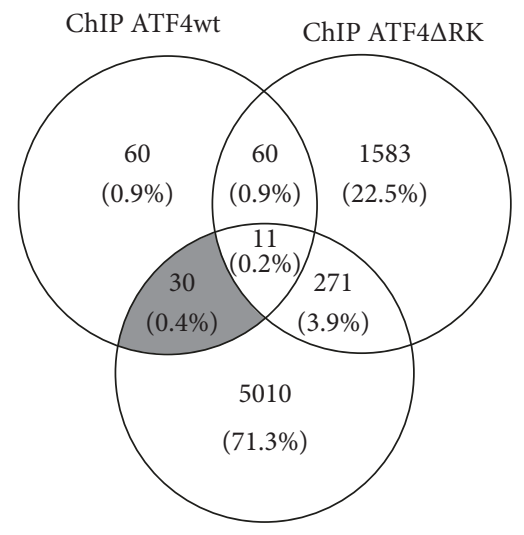

Array ATF4wt_S vs. ATF4wt

ChIP ATF4wt and Array ATF4wt_S vs. ATF4wt

endoplasmic reticulum unfolded protein response $(\mathrm{GO})$

CHOP-ATF3 complex (GO)

CHOP-C/EBP complex (GO)

(a)

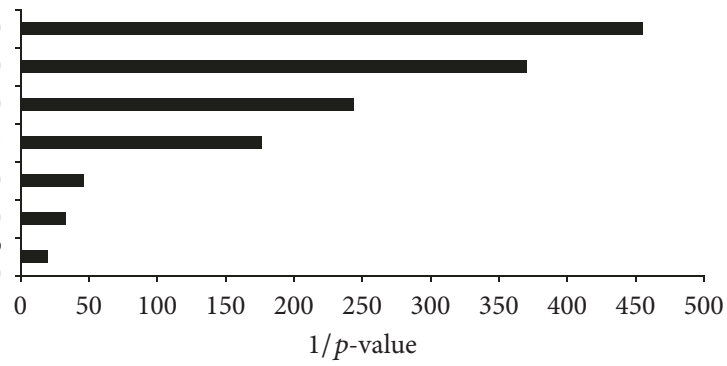

(c)

Figure 4: ChIP-on-chip Assay. (a) Heatmap representation comparing genes with a $-\log p$ value $>10$ expressed in both ATF4wt and ATF $4 \Delta$ RK samples. Upregulated genes in ATF4wt and ATF4 $\Delta$ RK are highlighted showing different pattern of expression. Color scale reflects expression level; black indicates no difference to control sample and green indicates increased expression compared to control. (b) Venn diagram depicting changes in genes of HL-1 cardiomyocytes overexpressing ATF4wt compared to HL-1 cardiomyocytes overexpressing ATF $4 \Delta$ RK. Numbers of ATF4wt and ATF4 $\Delta$ RK binding genes, with at least a fold change $\geq 1.2$ or $\leq 0.83$, and $p$ value $\leq 0.05$ compared to cells overexpressing GFP is indicated in circles (upper panel). For the grey area (genes enriched in ATF4wt but not in ATF4 RK), a gene ontology analysis was performed (lower panel). The main terms and pathways enriched only in the ATF4wt binding sites dataset are shown along with corresponding $p$ values. (c) Venn diagram depicting changes in ATF4 binding genes in ATF4wt and ATF4 $\Delta$ RK overexpressing cardiomyocytes in comparison to genes that are enriched in the stimulated cardiomyocytes overexpressing ATF4wt versus nonstimulated cardiomyocytes overexpressing ATF4wt microarray gene expression analysis. Numbers of genes, with at least a fold change $\geq 1.2$ or $\leq 0.83$, and $p$ value $\leq 0.05$ compared to cells overexpressing GFP are indicated in circles (upper panel). For the grey area (genes enriched in ATF4wt ChIP and stimulated cardiomyocytes overexpressing ATF4wt gene expression array only), a gene ontology analysis was performed (lower panel). The main terms and pathways are shown along with corresponding $p$ values. 


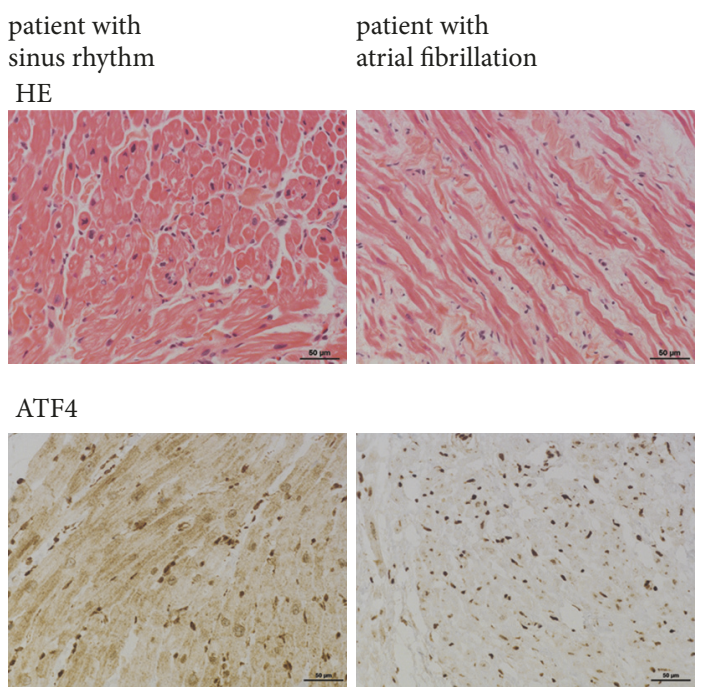

(a)

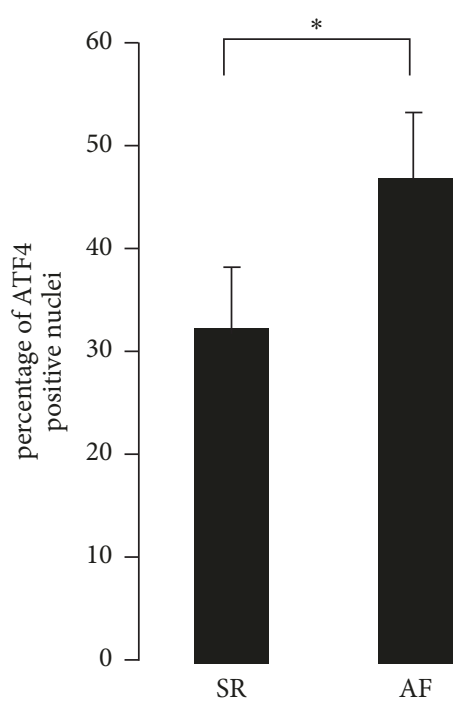

(b)

FIGURE 5: Immunohistological analysis of ATF4 expression in atrial tissue obtained from patients with sinus rhythm and with atrial fibrillation. (a) Representative images of HE staining and staining with antibodies directed against ATF4 (dark brown). Bar, $50 \mu \mathrm{m}$. (b) Percentage of ATF4 positive nuclei in histological specimen obtained from patients in sinus rhythm compared to patients with atrial fibrillation. $*$ indicates $p<0.05$.

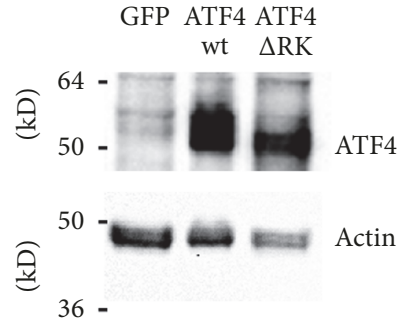

(a)

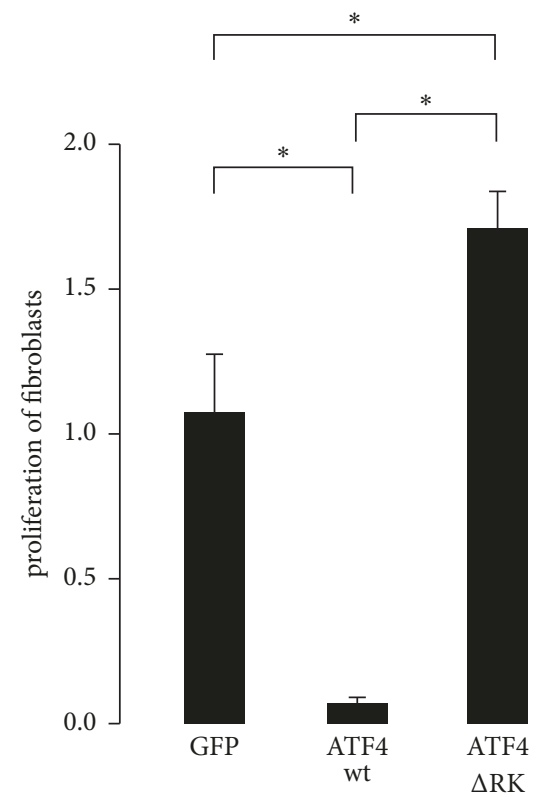

(b)

FIGURE 6: ATF4 overexpression in fibroblasts. (a) Representative western blots showing ATF4 expression in murine fibroblasts overexpressing GFP, ATF4wt, or ATF4 $\Delta$ RK. (b) Cell proliferation was assessed by BrdU assay $(n=4)$. $*$ indicates $p<0.05$. 
overexpressed cells, proliferation was increased compared to GFP overexpression (Figure 6(b)) suggesting a dominant negative effect on cardiac fibroblast proliferation.

\section{Discussion}

Solid evidence supports the hypothesis that atrial fibrillation is associated with both oxidative stress and endoplasmic reticulum stress in the atrial cardiomyocyte [27]. In fact, serum markers of oxidative stress have been shown to be elevated in AF patients [1]. Moreover, gene expression profiling in humans has revealed that $\mathrm{AF}$ is associated with a reduction of the expression of antioxidant genes and an increase in genes related to ROS suggesting that AF promotes a shift toward a prooxidant cell state in cardiomyocytes $[8,9]$. Oxidants can modify ion channel activity and induce highly specific and tightly regulated cell stress signaling pathways that promote atrial remodeling. ER stress in atrial fibrillation is less well characterized. However, a recent report has demonstrated that tachypacing-induced apoptosis in atrial cardiomyocytes is regulated by ER stress-mediated MAP and MAPKs [10]. Correspondingly, chemical inhibitors of ER stress were shown to be partially protective suggesting that endoplasmic reticulum signaling is important for atrial cardiomyocyte apoptosis and remodeling in AF and a potential target for therapy. Moreover, ER stress could emerge from changes in glucose metabolism that would affect $\mathrm{N}$-glycosylation in the ER and ER stress [28]. Indeed, diabetes seems to play an important role in the complex pathophysiology of atrial fibrillation.

Cardiomyocyte death is an important part of the atrial remodeling in response to atrial fibrillation. It is assumed that activation of specific cardiomyocyte prodeath transcription factors promotes the controlled demise of individual cardiomyocytes known as apoptosis. The current study provides insight into the transcription factors that regulate cardiomyocyte viability in cardiomyocytes that are exposed to atrial fibrillation. Specifically, we show that the transcription factor ATF4 is induced by both oxidative and ER stress. In consistence with the evidence that oxidative stress and ER stress are important components of the atrial cardiomyocyte remodeling process, we further demonstrate that AF induces ATF4 using a cellular model of atrial fibrillation. Moreover, forced expression of ATF4 sensitizes cardiomyocytes to cell death. Consistent with ATF4's role in regulating an upstream aspect of the cell stress induced death pathway, we found that ATF4 overexpression causes the induction of several apoptosis associated cell stress genes. Although known to be a stress-responsive protein, these results for the first time establish ATF4 as a protein that can be induced by tachypacing in cardiomyocytes and functions to lower the threshold for tachypacing induced death in cardiomyocytes.

ATF4 has an important role in regulating physiological responses to metabolic and redox processes and acts as a key transcription regulator of the integrated stress response (ISR) [6]. Classically, stress-mediated enhancement of ATF4 levels is known to occur via enhanced efficiency of translation of constant levels of ATF4 mRNA. Elevated levels of phosphorylated eukaryotic initiation translation factor 2 delay capacitation of reinitiating ribosomes, thereby fostering translation initiation at the ATF4 coding sequence, which subsequently permits ATF4 protein expression [11, 25]. Consistent with this model, we found an upregulation of ATF4 protein expression in tachypaced cardiomyocytes and cardiomyocytes exposed to ER stress and oxidative stress. In addition, we also observed an increase in ATF4 mRNA levels, thereby confirming reports describing a role for transcriptional regulation in ATF4 induction by different types of cell stress.

Originally described as a transcriptional repressor, ATF4 has been shown to have an activating effect on a number of several target genes, a significant part of which mediates cell stress responses and is involved in cell death and cell survival. Correspondingly, the gene array data presented in this study suggests that ATF4 acts at least in part as a transcriptional activator. Indeed, electrical stimulation in ATF4 overexpressing cardiomyocytes led to a pattern of gene expression characteristic of ER stress, oxidative stress, inflammation, and cell death suggesting that ATF4 can promote cardiomyocyte remodeling and cardiomyocyte death. Interestingly, ATF4 overexpression in unstimulated cardiomyocytes mainly influenced the expression of genes involved in amino acid biosynthesis suggesting that the effects of ATF4 expression are context specific.

Taken together, the data presented in this work are in support of a prodeath role of ATF4 in atrial cardiomyocytes. Generally, it is accepted that a short-lasting activation of the ISR is associated with an adaptive response in order to restore cellular homeostasis while a prolonged duration of ISR can cause the activation of prodeath pathways. ATF4 plays a key role in the switch between prodeath and prosurvival signaling by the ISR. In fact, previous findings regarding the roles of ATF4 in different tissues have yielded different results, and ATF4 is already known to have functions that are limited to a specific cell type. Specifically, ATF $4^{-/-}$fibroblasts are impaired in expressing genes involved in glutathione biosynthesis and resistance to oxidative stress [20]. However, ATF4 itself is capable of inducing cell death. In vitro studies in cortical neurons and an in vivo model of cerebral ischemia have yielded a prodeath role of ATF4 [29]. On the contrary, an elevated level of ATF4 expression has been shown in different cancer cell lines [30]. Different metabolic demands of individual cell types might help to explain the distinct role of ATF4 in cell death and survival. Moreover, differently expressed dimerization partners of ATF4 might also significantly impact its function. Interestingly, a recent study using mathematical modelling of the ISR has revealed that the ISR has three distinct activity states depending on the level and duration of stress. These activity states were associated with different outcomes with regard to cell death or survival [31]. Lamirault et al. [8] have carried out a detailed analysis of gene expression profiles associated with atrial fibrillation. Several of the genes that were regulated differently between patients in SR and AF include GATA4, glutathione peroxidase, and TNF. These genes have been linked to ER stress and ATF4 expression.

Histological analysis revealed a higher amount of ATF4 positive nuclei in AF patients compared to SR patients. However, the histological data must be interpreted with caution. 
Patients with mitral valve insufficiency are also at risk of AF even if in SR at time of surgery. The classification of sinus rhythm or atrial fibrillation was based on the available clinical data; however, documenting paroxysmal atrial fibrillation can be challenging in clinical practice. In fact, ATF4 mRNA levels did not differ significantly between the groups (data not shown). However, this finding does not necessarily argue against a prodeath role of ATF4 in atrial cardiomyocytes. ATF4 induced cell death might occur over a longer period of time, and ATF4 expression alone might not be sufficient to induce apoptosis in atrial cardiomyocytes in vivo. Moreover, surrounding fibroblasts and the intense chemical crosstalk between cardiomyocytes and cardiac fibroblast known to occur in cardiac tissue in vivo might also influence cell death/ survival decisions in cardiomyocytes expressing ATF4. Overexpression of ATF4 in cardiac fibroblasts caused a marked inhibition of fibroblast proliferation; however, ATF4 expression in fibroblasts was low in human atrial tissue specimen. In addition, inflammation seems to be important for atrial fibrillation. A recent report has shown that type I IFN response could act as a potential therapeutic target for postmyocardial infarction cardioprotection [32]. On the contrary, our gene expression analysis demonstrates a downregulation of interferon genes by ATF4, indicating again that the effects of ATF4 seem to be both cell-type and context specific.

4.1. Limitations. The study has a number of important limitations. Atrial fibrillation is a complex arrhythmia, which critically depends on the presence of substrate and triggers, and therefore cannot be completely mimicked by single cell experiments. HL-1 cells have been abundantly used for in vitro studies, but a mouse cell line cannot reflect the complex behavior of human cardiomyocytes in vivo. In fact, future research is necessary to precisely characterize upstream effects and effector pathways of ATF4. However, our results demonstrate that rapid field stimulation leads to an induction of ATF4 mRNA and increased ATF4 expression on the protein level and decreased cell viability. Indeed, a direct translation to atrial fibrillation cannot be done on the basis of the data. On the contrary, the expression of ATF4 in human atrial cardiomyocytes suggests that ATF4 could be one of the transcription factors that are important for atrial cardiomyocyte survival and apoptosis in atrial fibrillation. Therefore, further studies will be necessary to precisely characterize ATF4's dimerization partners in cardiomyocytes and its downstream signaling events.

\section{Conclusion}

In atrial cardiomyocytes, ATF4 is expressed in response to oxidative stress and ER stress. Accordingly, rapid pacing of cardiomyocytes is associated with ATF4 induction. Forced expression of ATF4 reduces cardiomyocyte viability. Additionally, ATF4 is expressed in human atrial cardiomyocytes in vivo. Despite the inherent limitations of an in vitro model of AF, the results presented in this study therefore support the notion that ATF4 could play an important role in the atrial remodeling in atrial fibrillation.

\author{
Abbreviation \\ ATF4: Activating transcription factor 4 \\ AF: Atrial fibrillation \\ bZIP: Basic leucine zipper \\ ER: Endoplasmic reticulum \\ MOI: Multiplicity of infection \\ ISR: Integrated stress response.
}

\section{Additional Points}

Highlights

(i) Here, we demonstrate the expression of the bZIP transcription factor ATF4 in human atrial cardiomyocytes.

(ii) In a cell culture based model of atrial fibrillation, ATF4 is induced by rapid pacing.

(iii) Forced expression of ATF4 reduces cell viability.

(iv) ATF4 binds to several promoters of cell stress genes and induces the expression of a number of ATF4 dependent cell stress genes.

\section{Conflicts of Interest}

The authors declare that there are no conflicts of interest regarding the publication of this article.

\section{Authors' Contributions}

Johanna K. Freundt, Philipp S. Lange, Gerrit Frommeyer, Andreas Huge, and Lars Eckardt designed the experiments and drafted the article. Johanna K. Freundt, Andreas Huge, and Philipp S. Lange acquired and analyzed data. Andreas Hoffmeier and Sven Martens provided the human tissue and designed the experiments. Fabian Wötzel performed the histological analysis. Andreas Huge and Johanna K. Freundt carried out the bioinformatics analysis. Gerrit Frommeyer, Andreas Huge, and Lars Eckardt revised the article critically for important intellectual content. All authors finally approved the version to be submitted.

\section{Acknowledgments}

The authors thank Sonja Schelhaas, Ph.D., and Michael Schäfers for their invaluable advice throughout this study. This work was supported by the Elisabeth und Rudolf Hirsch Stiftung für medizinische Forschung, Cologne, Germany (http://www.hirsch-stiftung.de/projekte.html). The authors also acknowledge support by Open Access Publication Fund of University of Muenster.

\section{Supplementary Materials}

Suppl. Figure S1: ATF4 expression in tunicamycin treated cardiomyocytes. Representative western blots showing protein expression of ATF4 in response to tunicamycin $(0,2$, and $4 \mu \mathrm{g} / \mathrm{ml}$ ) treatment. Suppl. Figure S2: original western blots. 
(A, B) Blots from Figure 2(a). (C, D) Blots from Figure 1(b). (E, F) Blots from Figure 1(c). (G, H) Blots from Figure 1(d). (I, J) Blots from Figure 6(a) (K, L). (Supplementary Materials)

\section{References}

[1] L. Fabritz, E. Guasch, C. Antoniades et al., "Expert consensus document: Defining the major health modifiers causing atrial fibrillation: A roadmap to underpin personalized prevention and treatment," Nature Reviews Cardiology, vol. 13, no. 4, pp. 230-237, 2016.

[2] S. Nattel, B. Burstein, and D. Dobrev, "Atrial remodeling and atrial fibrillation: mechanisms and implications.," Circulation: Arrhythmia and Electrophysiology, vol. 1, no. 1, pp. 62-73, 2008.

[3] S. Nattel and D. Dobrev, "Electrophysiological and molecular mechanisms of paroxysmal atrial fibrillation," Nature Reviews Cardiology, vol. 13, no. 10, pp. 1-16, 2016.

[4] M. S. Spach, J. F. Heidlage, P. C. Dolber, and R. C. Barr, "Mechanism of origin of conduction disturbances in aging human atrial bundles: Experimental and model study," Heart Rhythm, vol. 4, no. 2, pp. 175-185, 2007.

[5] J. Groenendyk, P. K. Sreenivasaiah, D. H. Kim, L. B. Agellon, and M. Michalak, "Biology of endoplasmic reticulum stress in the heart," Circulation Research, vol. 107, no. 10, pp. 1185-1197, 2010.

[6] K. Pakos-Zebrucka, I. Koryga, K. Mnich, M. Ljujic, A. Samali, and A. M. Gorman, "The integrated stress response," EMBO Reports, vol. 17, no. 10, pp. 1374-1395, 2016.

[7] N.-H. Kim, Y. Ahn, S. K. Oh et al., "Altered patterns of gene expression in response to chronic atrial fibrillation," International Heart Journal, vol. 46, no. 3, pp. 383-395, 2005.

[8] G. Lamirault, N. Gaborit, N. le Meur et al., "Gene expression profile associated with chronic atrial fibrillation and underlying valvular heart disease in man," Journal of Molecular and Cellular Cardiology, vol. 40, no. 1, pp. 173-184, 2006.

[9] R. Ohki, K. Yamamoto, S. Ueno et al., "Gene expression profiling of human atrial myocardium with atrial fibrillation by DNA microarray analysis," International Journal of Cardiology, vol. 102, no. 2, pp. 233-238, 2005.

[10] J. Shi, Q. Jiang, X. Ding, W. Xu, D. W. Wang, and M. Chen, "The ER stress-mediated mitochondrial apoptotic pathway and MAPKs modulate tachypacing-induced apoptosis in HL-1 atrial myocytes," PLoS ONE, vol. 10, no. 2, Article ID e0117567, 2015.

[11] K. Ameri and A. L. Harris, "Activating transcription factor 4," The International Journal of Biochemistry \& Cell Biology, vol. 40, no. 1, pp. 14-21, 2008.

[12] C. C. Glembotski, "The role of the unfolded protein response in the heart," Journal of Molecular and Cellular Cardiology, vol. 44, no. 3, pp. 453-459, 2008.

[13] P. D. Lu, H. P. Harding, and D. Ron, “Translation reinitiation at alternative open reading frames regulates gene expression in an integrated stress response," The Journal of Cell Biology, vol. 167, no. 1, pp. 27-33, 2004.

[14] H. Zinszner, M. Kuroda, X. Wang et al., "CHOP is implicated in programmed cell death in response to impaired function of the endoplasmic reticulum," Genes \& Development, vol. 12, no. 7, pp. 982-995, 1998.

[15] K. D. McCullough, J. L. Martindale, L. O. Klotz, T. Y. Aw, and N. J. Holbrook, "Gadd153 sensitizes cells to endoplasmic reticulum stress by down-regulating Bc12 and perturbing the cellular redox state," Molecular and Cellular Biology, vol. 21, no. 4, pp. 1249-1259, 2001.

[16] H. T. Adler, R. Chinery, D. Y. Wu et al., "Leukemic HRX fusion proteins inhibit GADD34-induced apoptosis and associate with the GADD34 and hSNF5/INI1 proteins," Molecular and Cellular Biology, vol. 19, no. 10, pp. 7050-7060, 1999.

[17] K. Du, S. Herzig, R. N. Kulkarni, and M. Montminy, “TRB3: a tribbles homolog that inhibits Akt/PKB activation by insulin in liver," Science, vol. 300, no. 5625, pp. 1574-1577, 2003.

[18] H. Puthalakath, L. A. O’Reilly, P. Gunn et al., "ER stress triggers apoptosis by activating BH3-only protein Bim," Cell, vol. 129, no. 7, pp. 1337-1349, 2007.

[19] C. C. Glembotski, "Endoplasmic reticulum stress in the heart," Circulation Research, vol. 101, no. 10, pp. 975-984, 2007.

[20] H. P. Harding, Y. Zhang, H. Zeng et al., "An integrated stress response regulates amino acid metabolism and resistance to oxidative stress," Molecular Cell, vol. 11, no. 3, pp. 619-633, 2003.

[21] B. J. J. M. Brundel, H. H. Kampinga, and R. H. Henning, "Calpain inhibition prevents pacing-induced cellular remodeling in a HL-1 myocyte model for atrial fibrillation," Cardiovascular Research, vol. 62, no. 3, pp. 521-528, 2004.

[22] Y.-H. Yeh, C.-T. Kuo, T.-H. Chan et al., “Transforming growth factor- $\beta$ and oxidative stress mediate tachycardia-induced cellular remodelling in cultured atrial-derived myocytes," Cardiovascular Research, vol. 91, no. 1, pp. 62-70, 2011.

[23] S. M. White, P. E. Constantin, and W. C. Claycomb, "Cardiac physiology at the cellular level: use of cultured HL-1 cardiomyocytes for studies of cardiac muscle cell structure and function," American Journal of Physiology-Heart and Circulatory Physiology, vol. 286, no. 3, pp. H823-H829, 2004.

[24] W. C. Claycomb, N. A. Lanson, B. S. Stallworth, D. B. Egeland, J. B. Delcarpio, A. Bahinski et al., "HL-1 cells: a cardiac muscle cell line that contracts and retains phenotypic characteristics of the adult cardiomyocyte," Proceedings of the National Academy of Sciences of the United States of America, vol. 95, pp. 2979-2984, 1998.

[25] P. S. Lange, J. C. Chavez, J. T. Pinto et al., "ATF4 is an oxidative stress-inducible, prodeath transcription factor in neurons in vitro and in vivo," The Journal of Experimental Medicine, vol. 205, no. 5, pp. 1227-1242, 2008.

[26] K. M. Vattem and R. C. Wek, "Reinitiation involving upstream ORFs regulates ATF4 mRNA translation in mammalian cells," Proceedings of the National Academy of Sciences of the United States of America, vol. 101, pp. 11269--11274, 2004.

[27] Y. Yeh, C. Kuo, T. Chan et al., "Transforming growth factor$\beta$ and oxidative stress mediate tachycardia-induced cellular remodelling in cultured atrial-derived myocytes," Cardiovascular Research, vol. 91, no. 1, pp. 62-70, 2011.

[28] M. Hano, L. Tomášová, M. Šereš, L. Pavlíková, A. Breier, and Z. Sulová, "Interplay between P-Glycoprotein Expression and Resistance to Endoplasmic Reticulum Stressors," Molecules, vol. 23, no. 2, p. 337, 2018.

[29] T. Hayashi, A. Saito, S. Okuno, M. Ferrand-Drake, R. L. Dodd, and P. H. Chan, "Damage to the endoplasmic reticulum and activation of apoptotic machinery by oxidative stress in ischemic neurons," Journal of Cerebral Blood Flow \& Metabolism, vol. 25, no. 1, pp. 41-53, 2005.

[30] M. Tanabe, H. Izumi, T. Ise et al., "Activating Transcription Factor 4 Increases the Cisplatin Resistance of Human Cancer Cell Lines," Cancer Research, vol. 63, no. 24, pp. 8592-8595, 2003. 
[31] K. Erguler, M. Pieri, and C. Deltas, "A mathematical model of the unfolded protein stress response reveals the decision mechanism for recovery, adaptation and apoptosis," BMC Systems Biology, vol. 7, no. 16, 2013.

[32] K. R. King, A. D. Aguirre, Y.-X. Ye et al., "IRF3 and type I interferons fuel a fatal response to myocardial infarction," Nature Medicine, vol. 23, no. 12, pp. 1481-1487, 2017. 


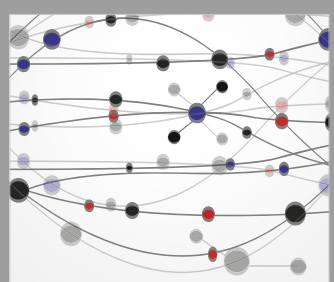

The Scientific World Journal
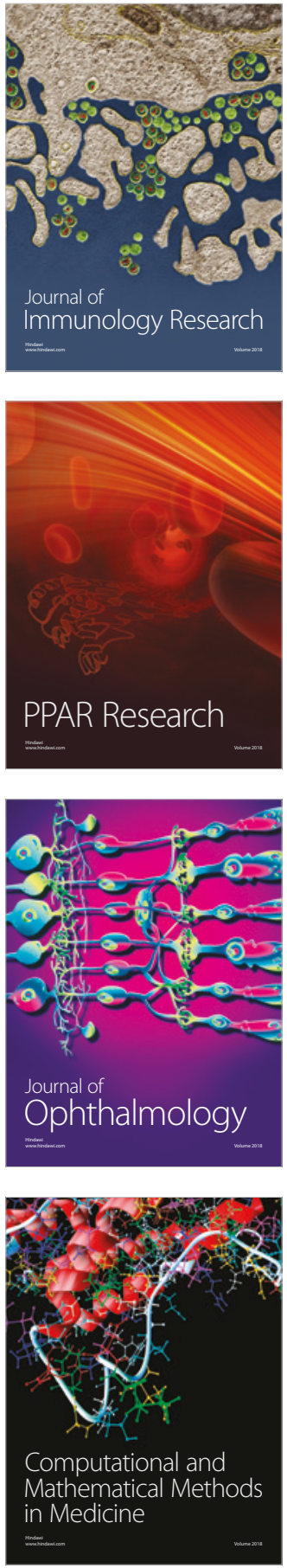

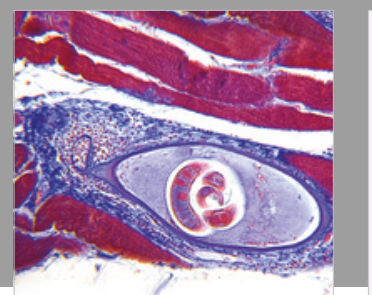

Gastroenterology Research and Practice

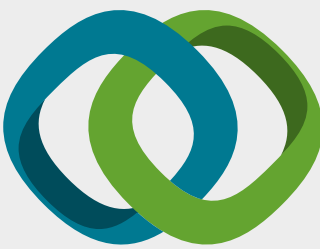

\section{Hindawi}

Submit your manuscripts at

www.hindawi.com
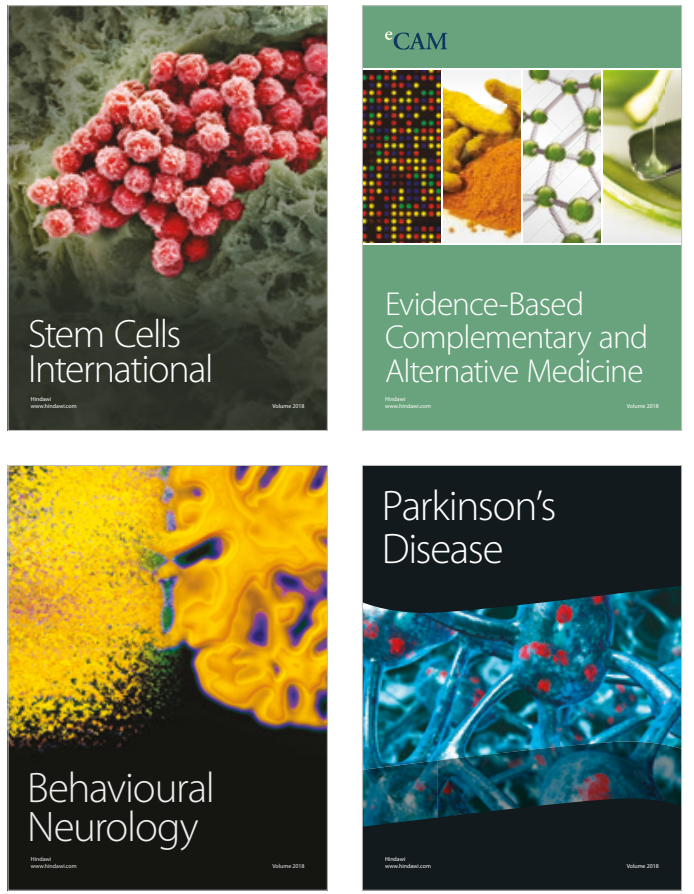

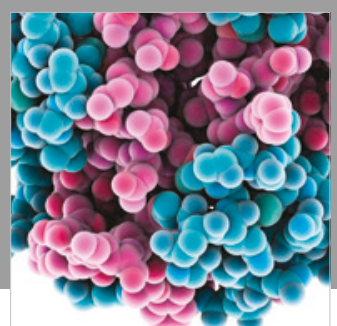

ournal of

Diabetes Research

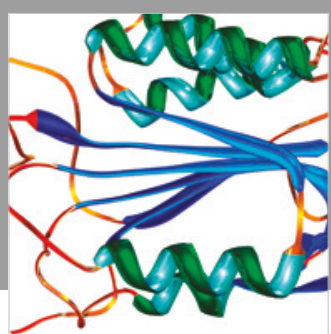

Disease Markers
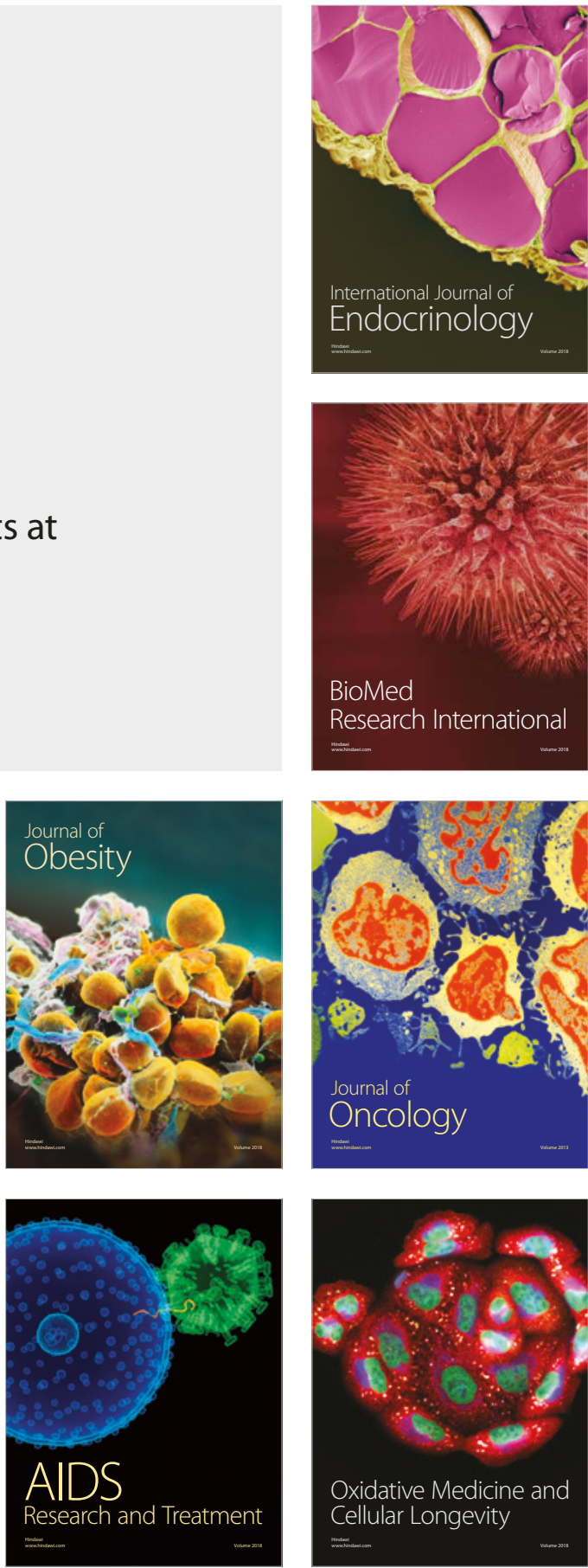